\title{
On the Existence of Solutions to Nonlinear Equations Involving Singular Mappings with Non-zero $p$-Kernel
}

\author{
Agnieszka Prusińska • Alexey A. Tret'yakov
}

Received: 18 May 2010 / Accepted: 22 March 2011 /

Published online: 13 April 2011

(C) The Author(s) 2011. This article is published with open access at Springerlink.com

\begin{abstract}
The paper presents the continuation of the previous results devoted to the problem of solutions existence to nonlinear equations in singular case where a linear part of considered mapping determining the equation may be degenerate at the corresponding initial point. We study the case when the $p$-kernel of the mapping is non trivial. Such type of problems appears in various mathematical models and applications. The $p$-regularity theory is used in our analysis and some concepts and technics of set-valued approach.
\end{abstract}

Keywords $p$-regularity $\cdot$ Singularity $\cdot$ Nonlinear mapping $\cdot$ Multimapping • Contraction $\cdot$ Factor operator $\cdot p$-kernel

Mathematics Subject Classifications (2010) $47 \mathrm{H} 10$ • 47A50 • 47J05 • 47J25

\section{Introduction}

In this paper we continue consideration of the solution existence problem to the nonlinear equation which was studied in [15].

\footnotetext{
A. Prusińska $(\varangle) \cdot$ A. A. Tret'yakov Institute of Mathematics and Physics, Siedlce University of Natural Sciences and Humanities, 3-go Maja 54, 08-110 Siedlce, Poland e-mail: aprus@ap.siedlce.pl
}

\section{A. A. Tret'yakov}

System Research Institute, Polish Academy of Sciences, Newelska 6, 01-447 Warsaw, Poland e-mail: tret@ap.siedlce.pl

A. A. Tret'yakov

Dorodnicyn Computing Center, Russian Academy of Sciences,

Vavilova 40, Moscow, Russia 
Let us consider the following nonlinear equation:

$$
F(x)=0,
$$

where $F: X \rightarrow Y$ and $X, Y$ are Banach spaces. There are many results on solution existence of (1) in non-degenerate case, i.e. when in some point $x_{0}$ we have $\operatorname{Im} F^{\prime}\left(x_{0}\right)=Y$ (see [9]). But in degenerate case such results either don't exist or at least there are not unified approach for analysis of such type problems.

In present paper in the main result (Theorem 2) we give sufficient conditions for existence of local solutions to some equations in non-regular (singular) case when $p$-kernel of $p$ th order derivative of $F$ at the initial point $x_{0}$ is nonempty. Earlier we have considered the trivial $p$-kernel case (see [15]). This result can be applied for instance to the following nonlinear boundary value problem:

$$
F(u)=\Delta u-(\varepsilon+\bar{\varepsilon}) g(u)=0 \quad \text { in } \Omega
$$

with the boundary conditions

$$
u=0 \quad \text { on } \partial \Omega \text {, }
$$

where $\Omega$ is a bounded region in $\mathbb{R}^{n}$ with smooth boundary, $\Delta$ is Laplacian, $u$ belongs to a suitable Banach space (let say $H_{0}^{s+2}$, such that $F: H_{0}^{s+2} \times \mathbb{R} \rightarrow H^{s}$ ), $\bar{\varepsilon}$ is an eigenvalue of $\Delta, g(0)=0, g^{\prime}(0)=1, \varepsilon$ is sufficiently small number and $H^{s}$ is Hilbert space of $s$-times continuously differentiable functions with traditional scalar product.

In this case at the initial point $u_{0}=0$ operator $F^{\prime}\left(u_{0}\right)$ is singular and (as we will show later) 2-kernel of $F^{\prime \prime}\left(u_{0}\right)$ is nonempty.

Another example is the following integral equation

$$
\begin{aligned}
F(x)= & x(t)-\frac{1}{\sqrt{\pi}} \varepsilon \cos t-\frac{1}{\pi} \int_{0}^{2 \pi} \cos (t-\tau) x(\tau) d \tau \\
& -\frac{1}{\sqrt{\pi}} \sin t \int_{0}^{2 \pi} \sin \tau \cdot \cos \tau(x(\tau))^{2} d \tau, \quad t \in[0,2 \pi]
\end{aligned}
$$

where $\varepsilon \in \mathbb{R}$ is a small parameter, $F: X \rightarrow X, X=L_{2}[0,1]$.

The problem is the solution existence to the above equation for $\varepsilon \in \mathbb{R}$ sufficiently small. In this case at the initial point $x_{0}=0, F^{\prime}\left(x_{0}\right)$ is singular and 2-kernel of $F^{\prime \prime}\left(x_{0}\right)$ is nonempty similarly like in Eq. 2. We consider this example in the final part of our paper as well.

Let us recall some definitions and denotations.

The problem (1) is called regular at $x_{0}$ if $\operatorname{Im} F^{\prime}\left(x_{0}\right)=Y$. Otherwise, the problem (1) is called nonregular (singular or degenerate) at $x_{0}$.

We use the $p$-regularity theory (see e.g. [2-7, 11, 13, 16-19]) for description and investigation of solutions existence in the degenerate case.

Let $p$ be a natural number and let $B: X \times \ldots \times X \rightarrow Y$ be a continuous $p$ multilinear mapping. A $p$-form associated to $B$ is the map $B[\cdot]^{p}: X \rightarrow Y$ defined by

$$
B[x]^{p}=B(\underbrace{x, \ldots, x}_{p})
$$

for $x \in X$. Alternatively, we may simply view $B[\cdot]^{p}$ as homogeneous polynomial map $B: X \rightarrow Y$ of degree $p$, i.e. $B(\alpha x)=\alpha^{p} \cdot B(x)$. 
Throughout this paper we assume that the mapping $F: X \rightarrow Y$ is continuously $p$-times Fréchet differentiable on $X$ and write $F \in \mathcal{C}^{p}(X)$. Its $p$-th order derivative at $x \in X$ we denote as usual by $F^{(p)}(x)$ (a symmetric multilinear map of $p$ copies of $X$ to $Y$ ) and the associated $p$-form is

$$
F^{(p)}(x)[h]^{p}=F^{(p)}(x)[\underbrace{h, \ldots, h}_{p}] .
$$

Furthermore, we use the following notation for the $p$-kernel of the mapping $F^{(p)}(x)$ (zero locus of $F^{(p)}(x)$ )

$$
\operatorname{Ker}^{p} F^{(p)}(x)=\left\{h \in X: F^{(p)}(x)[h]^{p}=0\right\} .
$$

Denote also by $\mathcal{L}(X, Y)$ a space of all continuous linear operators from $X$ to $Y$. The set

$$
M\left(x^{*}\right)=\left\{x \in U: F(x)=F\left(x^{*}\right)=0\right\},
$$

is called the solution set for the mapping $F$ in neighborhood $U \subseteq X$.

We call $h$ a tangent vector to a set $M \subseteq X$ at $x^{*} \in M$ if there exist $\varepsilon>0$ sufficiently small and a map $r:[0, \varepsilon] \rightarrow X$ with the property that for $t \in[0, \varepsilon]$, we have $x^{*}+t h+r(t) \in M$ and $\|r(t)\|=o(t)$. The collection of all tangent vectors at $x^{*}$ is called the tangent cone to $M$ at $x^{*}$ and it is denoted by $T_{1} M\left(x^{*}\right)$ (see e.g. [1]).

By the mapping $\Phi: X \rightarrow 2^{Y}$ we mean multimapping (or a multivalued mapping) from $X$ to the collection of all subsets of $Y$.

For a linear operator $\Lambda: X \rightarrow Y$ we denote by $\Lambda^{-1}$ its right inverse, that is $\Lambda^{-1}: Y \rightarrow 2^{X}$ which maps any element $y \in Y$ on its complete inverse image of the mapping $\Lambda, \Lambda^{-1} y=\{x \in X: \Lambda x=y\}$, and of course $\Lambda \Lambda^{-1}=I_{Y}$.

By the "norm" of such right inverse operator we mean the number

$$
\left\|\Lambda^{-1}\right\|=\sup _{\|y\|=1} \inf \{\|x\|: \Lambda x=y, x \in X\} .
$$

Note that if $\Lambda$ is one-to-one, then $\left\|\Lambda^{-1}\right\|$ can be considered as the usual norm of the element $\Lambda^{-1}$ in the space $\mathcal{L}(Y, X)$.

In our further considerations, by $\Lambda^{-1}$ we shall mean just right inverse operator (multivalued) with the norm defined by (4).

\section{Elements of $p$-Regularity Theory}

Let $F: X \rightarrow Y$ is $p$-times Fréchet differentiable mapping. If $F^{(i)}\left(x_{0}\right)=0$, where $i=1, \ldots, p-1$, then we say that $F$ is completely degenerate at $x_{0}$ up to the order $p$.

In this paper we consider the case when the regularity condition does not hold, i.e. $\operatorname{Im} F^{\prime}\left(x_{0}\right) \neq Y$, but the mapping $F$ is $p$-regular. First of all, let us remind the definition of $p$-regularity and construction of $p$-factor operator.

Consider a sufficiently smooth nonlinear mapping $F: X \rightarrow Y$. We construct the $p$-factor operator under the assumption that $Y$ is decomposed into a direct sum

$$
Y=Y_{1} \oplus \ldots \oplus Y_{p},
$$

where $Y_{1}=\overline{\operatorname{Im} F^{\prime}\left(x_{0}\right)}$ (the closure of the image of the first derivative of $F$ evaluated at $x_{0}$ ), and the remaining spaces are defined as follows. Let $Z_{1}=Y, Z_{2}$ be closed 
complementary subspace to $Y_{1}$ (we are assuming that such closed complement exists), and let $P_{Z_{2}}: Y \rightarrow Z_{2}$ be the projection operator onto $Z_{2}$ along $Y_{1}$. Let $Y_{2}$ be the closed linear span of the image of the quadratic map $P_{Z_{2}} F^{(2)}\left(x_{0}\right)[\cdot]^{2}$. More generally, define inductively,

$$
Y_{i}=\overline{\operatorname{span}} \operatorname{Im} P_{Z_{i}} F^{(i)}\left(x_{0}\right)[\cdot]^{i} \subseteq Z_{i}, \quad i=2, \ldots, p-1,
$$

where $Z_{i}$ is a choice of closed complementary subspace for $\left(Y_{1} \oplus \ldots \oplus Y_{i-1}\right)$ with respect to $Y, i=2, \ldots, p$ and $P_{Z_{i}}: Y \rightarrow Z_{i}$ is the projection operator onto $Z_{i}$ along $\left(Y_{1} \oplus \ldots \oplus Y_{i-1}\right)$ with respect to $Y, i=2, \ldots, p$. Finally, $Y_{p}=Z_{p}$. The order $p$ is chosen as the minimum number for which (4) holds. Now, define the following mappings (see [11,15,19]), $f_{i}: U \rightarrow Y_{i}, f_{i}(x)=P_{Y_{i}} F(x)$, $i=1, \ldots, p$, where $P_{Y_{i}}: Y \rightarrow Y_{i}$ is the projection operator onto $Y_{i}$ along $\left(Y_{1} \oplus \ldots \oplus Y_{i-1} \oplus Y_{i+1} \oplus \ldots \oplus Y_{p}\right)$ with respect to $Y, i=1, \ldots, p$.

Definition 1 The linear operator $\Lambda_{h} \in \mathcal{L}\left(X, Y_{1} \oplus \ldots \oplus Y_{p}\right)$ is defined for some $h \in X$ by

$$
\Lambda_{h}(x)=f_{1}^{\prime}\left(x_{0}\right)[x]+f_{2}^{\prime \prime}\left(x_{0}\right)[h, x]+\ldots+\frac{1}{(p-1) !} f_{p}^{(p)}\left(x_{0}\right)[h, \ldots, h, x], \quad x \in X
$$

and is called the p-factor operator.

We will also use more exact denotation $\Lambda_{h}=\left(\Lambda_{h, 1}+\Lambda_{h, 2}+\ldots+\Lambda_{h, p}\right)$, where $\Lambda_{h, 1}=\frac{1}{(k-1) !} f_{k}^{(k)}\left(x_{0}\right)[h]^{k-1}$.

Sometimes it is convenient to use the following equivalent definition of $p$-factor operator $\tilde{\Lambda}_{h} \in \mathcal{L}\left(X, Y_{1} \times \ldots \times Y_{p}\right)$ for some $h \in X$,

$$
\widetilde{\Lambda}_{h}(x)=\left(f_{1}^{\prime}\left(x_{0}\right)[x], f_{2}^{\prime \prime}\left(x_{0}\right)[h, x], \ldots, \frac{1}{(p-1) !} f_{p}^{(p)}\left(x_{0}\right)[h, \ldots, h, x]\right), \quad x \in X .
$$

Note that in completely degenerate case the $p$-factor operator has the form $\frac{1}{(p-1) !} F^{(p)}\left(x_{0}\right)[h]^{p-1}$.

In other words, we construct a decomposition of "non-regular part" of the mapping $F$ on partial mappings $f_{i}$ in such a way that all of these mappings are completely degenerate up to the order $i-1$ where $i=2, \ldots, p$.

For our further considerations we need the following generalization of the notion of regular mapping.

Definition 2 We say that the mapping $F$ is $p$-regular at $x_{0}$ along $h$ if

$$
\operatorname{Im} \Lambda_{h}=Y .
$$

Let us introduce corresponding nonlinear operator

$$
\Psi[x]^{p}=f_{1}^{\prime}\left(x_{0}\right)[x]+f_{2}^{\prime \prime}\left(x_{0}\right)[x]^{2}+\ldots+f_{p}^{(p)}\left(x_{0}\right)[x]^{p}
$$

and

$$
\operatorname{Ker}^{p} \Psi[x]^{p}=\left\{x: f_{1}^{\prime}\left(x_{0}\right)[x]+f_{2}^{\prime \prime}\left(x_{0}\right)[x]^{2}+\ldots+f_{p}^{(p)}\left(x_{0}\right)[x]^{p}=0\right\} .
$$

It is easy to see that $\Psi[h]^{p}=\Lambda_{h, 1}(h)+\Lambda_{h, 2}(h)+\ldots+(p-1) ! \Lambda_{h, p}(h)$. 
Definition 3 We say that the mapping $F$ is $p$-regular at $x_{0}$ if either it is $p$-regular along every $h$ belonging to the set

$$
\operatorname{Ker}^{p} \Psi_{p}[x]^{p} \backslash\{0\}
$$

or $\operatorname{Ker}^{p} \Psi_{p}[x]^{p}=\{0\}$.

Remark 1 Sets $\operatorname{Ker}^{p} \Psi_{p}[x]^{p}$, and $\bigcap_{k=1}^{p} \operatorname{Ker}^{k} f_{k}^{(k)}\left(x_{0}\right)$ coincide. It follows from the definitions of $\operatorname{Ker}^{p} \Psi[x]^{p}$ and $f_{k}(x), k=1, \ldots, p$.

Let us consider some examples that illustrate the above introduced construction of $p$-regularity.

Example 1 Consider a type of (2) equation

$$
F(u, \varepsilon)=\Delta u-(\varepsilon-10) g(u)=0
$$

on $\Omega=[0, \pi] \times[0, \pi]$ in $\mathbb{R}^{2}$ with $u=0$ on $\partial \Omega, \bar{\varepsilon}=-10$. Assume that $g(0)=0$, $g^{\prime}(0)=1, g^{\prime \prime}(0)=1$.

We would like to find out whether the mapping $F(u, \varepsilon)$ is $p$-regular $(p=2)$ at the point $x_{0}=\left(u_{0}, \varepsilon_{0}\right)=(0,0)$ along some $h=\left(h_{u}, h_{\varepsilon}\right) \in \operatorname{Ker} F^{\prime}(0,0) \cap \operatorname{Ker}^{2} P_{2} F^{\prime \prime}(0,0)$. To answer this question let us formulate the following result that gives sufficient conditions for $p$-regularity of mapping $F(u, \varepsilon)$.

Consider a general equation (2) for $p=2$, i.e.

$$
F(u, \varepsilon)=\Delta u-(\varepsilon+\bar{\varepsilon}) g(u),
$$

$F: H_{0}^{s+2} \times \mathbb{R} \rightarrow H^{s}, \quad g \in \mathcal{C}^{p+1}, u=u(y), y \in \mathbb{R}^{m}$ and $\bar{\varepsilon}$ is an eigenvalue of $\Delta$ with multiplicity $l>1$. Here $g(0)=0, g^{\prime}(0)=1, g^{\prime \prime}(0)=1, p=2$. Let us give some auxiliary assumptions and denotations that will be necessary for proving $p$-regularity property of mapping $F(x)$.

Let $F_{u}^{\prime}(0,0)$ be a Fredholm operator and $\operatorname{Ker} F^{\prime}(0,0)=\operatorname{Ker}(\Delta-\bar{\varepsilon} I)$ be spanned by the orthogonal functions $u_{1}, \ldots, u_{l}$ from $\mathcal{L}_{2}$. Consider an element

$$
u=z_{1} u_{1}+\ldots+z_{l} u_{l}, \quad z_{i} \in \mathbb{R}, i=1, \ldots, l .
$$

Since $g(0)=0$, we obtain $Y_{1}=\operatorname{Im} F_{x}^{\prime}(0)=\operatorname{Im} F_{u}^{\prime}(0), Y_{2}=\left(\operatorname{Im} F_{x}^{\prime}(0)\right)^{\perp}=\left(\operatorname{Im} F_{u}^{\prime}(0)\right)^{\perp}$, and $H^{s}=Y_{1} \oplus Y_{2}$. For Fredholm operator $F_{u}^{\prime}(0,0)$ we have $\operatorname{Ker}(\Delta-\bar{\varepsilon} I)=\left(\operatorname{Im} F_{u}^{\prime}(0)\right)^{\perp}, F^{\prime \prime}(0)[u, \varepsilon]^{2}=-\bar{\varepsilon} g^{\prime \prime}(0) u^{2}-2 u \varepsilon=-\bar{\varepsilon} u^{2}-2 u \varepsilon$.

Define $z=\left(z_{1}, \ldots, z_{l}\right), h=\left(h_{u}, h_{\varepsilon}\right)=\left(z_{1} u_{1}+\ldots+z_{l} u_{l}, \varepsilon\right)$ and

$$
\begin{aligned}
Q[h]^{2} & =P_{2} F^{\prime \prime}(0)[h]^{2}=\left(\left\langle F^{\prime \prime}(0)[h]^{2}, u_{1}\right\rangle, \ldots,\left\langle F^{\prime \prime}(0)[h]^{2}, u_{l}\right\rangle\right) \\
& =\left(Q_{1}[h]^{2}, \ldots, Q_{l}[h]^{2}\right),
\end{aligned}
$$

where

$$
\begin{aligned}
Q_{i}[h]^{2} & =Q_{i}[z, \varepsilon]^{2}=\left\langle F^{\prime \prime}(0)[z, \varepsilon]^{2}, u_{i}\right\rangle \\
& =2 \varepsilon z_{i}+\bar{\varepsilon} \int_{\Omega} u_{i}\left(z_{1} u_{1}+\ldots+z_{l} u_{l}\right)^{2} d y \\
& =2 \varepsilon z_{i}+\sum_{j, k} C_{i}^{j k} z_{j} z_{k},
\end{aligned}
$$


$i=1, \ldots, l$ where $C_{i}^{j k}$ are so called branching coefficients such that $C_{i}^{j k}=\int_{\Omega} u_{j} u_{k} u_{i} d y, j, k, i=1, \ldots, l$.

Let us introduce $M(z)=M\left(z_{1}, \ldots, z_{l}\right)$, the $l \times l$ symmetric matrix, where $(i j)$ entry is

$$
\sum_{k=1}^{l}\left(\int_{\Omega} u_{i} u_{j} u_{k} d y\right) z_{k}
$$

and let

$$
\begin{aligned}
\operatorname{det}(M(z)-\varepsilon I) & =D_{1}(\varepsilon)=\varepsilon^{l}+A_{1} \varepsilon^{l-1}+\ldots+A_{l}, \\
\operatorname{det}(M(z)-2 \varepsilon I) & =D_{2}(\varepsilon)=2^{l} \varepsilon^{l}+2^{l-1} A_{1} \varepsilon^{l-1}+\ldots+A_{l},
\end{aligned}
$$

where $A_{i}$ is an homogeneous polynomial in the variables $z_{1}, \ldots, z_{l}$ of degree $i$. Let $R\left(D_{1}, D_{2}\right)$ be a resultant of $D_{1}(\varepsilon)$ and $D_{2}(\varepsilon)$ and

$$
R\left(D_{1}, D_{2}\right)=\operatorname{det}\left(\begin{array}{cccccccc}
1 & A_{1} & A_{2} & \ldots & A_{l} & 0 & \cdots & 0 \\
0 & 1 & A_{1} & \cdots & A_{l-1} & A_{l} & \cdots & 0 \\
\cdots & \cdots & \cdots & \cdots & \cdots & \cdots & \cdots & \ldots \\
0 & \cdots & \cdots & \cdots & 1 & A_{1} & \cdots & A_{l} \\
2^{l} & 2^{l-1} A_{1} & 2^{l-2} A_{2} & \ldots & A_{l} & 0 & \cdots & 0 \\
0 & 2^{l} & 2^{l-1} A_{1} & 2^{l-2} A_{2} & \cdots & A_{l} & \cdots & 0 \\
\cdots & \ldots & \ldots & \cdots & \cdots & \cdots & \cdots & \cdots \\
0 & \cdots & \cdots & 0 & 2^{l} & 2^{l-1} A_{1} & \cdots & A_{l}
\end{array}\right)
$$

Denote $\bar{D}_{2}(\varepsilon)=\left(2^{l}-1\right) \varepsilon^{l-1}+\left(2^{l-1}-1\right) A_{1} \varepsilon^{l-2}+\ldots+A_{l-1}$.

We formulate sufficient conditions of 2-regularity of the mapping $F(x)$.

Theorem 1 Let $(z, \varepsilon) \in \operatorname{Ker}^{2} P_{2} F^{\prime \prime}(0)$ (equivalently $\left.(z, \varepsilon) \in \operatorname{Ker}^{2} Q\right)$ and $(z, \varepsilon) \neq 0$. Under the above assumptions and notations for general equation (2) we have:

If $\varepsilon \neq 0$ and $R\left(D_{1}, D_{2}\right) \neq 0$, then $F(x)$ is 2-regular along $(z, \varepsilon)$ at $(0,0)$.

If $\varepsilon=0$ and $\operatorname{Rank} M(z)=l-1$ then $F(x)$ is 2-regular along $(z, \varepsilon)$ at $(0,0)$.

Proof Consider the case $\varepsilon \neq 0$. Property of 2-regularity along $(z, \varepsilon)$ means that $l \times(l+1)$ matrix $(M(z)+2 \varepsilon I, 2 I z)$ must be nonsingular for

$$
h=(z, \varepsilon) \in \operatorname{Ker}^{2} P_{2} F^{\prime \prime}(0)=\operatorname{Ker}^{2} Q .
$$

It means that $\varepsilon$ can not be an eigenvalue of $M(z)$.

Element $(z, \varepsilon)$ is in $\operatorname{Ker}^{2} Q$ if and only if either $z$ is an eigenvector of $M(z)$ corresponding to the eigenvalue $2 \varepsilon$ or $z=(0, \ldots, 0)$. At $(0, \ldots, 0, \varepsilon), \varepsilon \neq 0, Q$ is always nonsingular (and of course, $R\left(D_{1}, \bar{D}_{2}\right) \neq 0$ ). At any $(z, \varepsilon) \in \operatorname{Ker}^{2} Q, Q$ is 2-regular along $(z, \varepsilon)$ if $\varepsilon$ is not an eigenvalue of $M(z)$. It follows that a sufficient conditions for 2-regularity along $(z, \varepsilon)$ is that neither $\varepsilon$, nor $2 \varepsilon$ are eigenvalue of the matrix $M(z)$. 
It means that polynomial

$$
\begin{gathered}
\varepsilon^{l}+A_{1} \varepsilon^{l-1}+\ldots+A_{l} \\
\left(2^{l-1}-1\right) \varepsilon^{l-1}+\left(2^{l-2}-1\right) A_{1} \varepsilon^{l-2}+\ldots+A_{l-1}
\end{gathered}
$$

does not have a common roots. This condition is equivalent to non-vanishing of the determinant of (8), which is equivalent to $R\left(D_{1}, \bar{D}_{2}\right) \neq 0$ for $\varepsilon \neq 0$.

The case $\varepsilon=0$ is simpler. For 2-regularity along $(z, 0)$ it is sufficient that the matrix $(M(z), I z)$ is not singular for $(z, 0)$ such that $(M(z), I z)(z, 0)^{\perp}=0$.

It means that either $\operatorname{dim} \operatorname{Ker}(M(z), I z)=1$ and we obtain Rank $M(z)=l-1$, or $\operatorname{dim} \operatorname{Ker}(M(z), I z)=0$ and $(z, 0) \notin \operatorname{Ker}^{2} Q$. This contradiction completes the proof.

Let us return to (6). We take eigenfunctions $u_{1}=\sin 3 y_{1} \sin y_{2}$, $u_{2}=\sin y_{1} \sin 3 y_{2}$ corresponding to eigenvalue $\bar{\varepsilon}=-10$ and $a=\int_{\Omega} u_{1}^{3} d y=\int_{\Omega} u_{2}^{3} d y=\frac{16}{27}$, $b=\int_{\Omega} u_{1}^{2} u_{2} d y=\int_{\Omega} u_{1} u_{2}^{2} d y=\frac{68}{75}$. Then

$$
P_{2} F^{\prime \prime}(0,0)[z, \varepsilon]^{2}=10\left(a z_{1}^{2}+2 z_{1} z_{2} b+z_{2}^{2} b+2 \varepsilon z_{1}, b z_{1}^{2}+2 z_{1} z_{2} b+a z_{2}^{2}+2 \varepsilon z_{2}\right)
$$

$$
M(z)=\left(\begin{array}{ll}
a z_{1}+b z_{2} & b z_{2}+b z_{1} \\
b z_{2}+b z_{1} & b z_{1}+a z_{2}
\end{array}\right) .
$$

From calculation of resultant (8) we have the following expression

$$
-2\left((a+b) z_{1}+(a+b) z_{2}\right)^{2}+9\left(\left(a z_{1}+b z_{2}\right)\left(b z_{1}+a z_{2}\right)-\left(b z_{1}+a z_{2}\right)^{2}\right)
$$

which can be rewritten as $A z_{1}^{2}+2 B z_{1} z_{2}+C z_{2}^{2}$, where $A C-B^{2}>0$, $A C=\left(2 a-5 a b+11 b^{2}\right)^{2}, \quad B^{2}=\frac{1}{4}\left(13 b^{2}-5 a^{2}+8 a b\right)^{2}$ for $a=16 / 27, b=68 / 75$. It means that $R\left(D_{1}, \bar{D}_{2}\right) \neq 0$ and in accordance to Theorem 1 , the mapping $F$ is 2-regular along $(z(\varepsilon), \varepsilon) \in \operatorname{Ker}^{2} Q, \varepsilon \neq 0$.

Example 2 Let us consider some modification of Eq. 2

$$
F(u)=\Delta u-\bar{\varepsilon} g(u)-\varepsilon \varphi(y)=0,
$$

where $\varphi \in H^{s}, \Omega=[0, \pi] \times[0, \pi], m=2, g(0)=0, g^{\prime}(0)=1, g^{\prime \prime}(0)=1, \bar{\varepsilon}$ is an eigenvalue of $\Delta, \varepsilon \in \mathbb{R}_{+}$a sufficiently small parameter and $\|\varphi(y)\|=1$. Similarly to how it was shown in previous example the mapping $F(u)$ is 2-regular at $u_{0}=0$ along some $z \in \operatorname{Ker}^{2} P_{2} F_{u}^{\prime \prime}(0)$, where $z=\bar{z}_{1} u_{1}+\bar{z}_{2} u_{2}$,

$$
P_{2} F_{u}^{\prime \prime}(0)[z]^{2}=10\left(2 z_{1}^{2}+2 z_{1} z_{2}+z_{2}, z_{1}^{2}+2 z_{1} z_{2}+z_{2}^{2}\right)
$$

if matrix $M(z)$ has $\operatorname{Rank} M(z)=l$ and $z$ is a solution of

$$
P_{2} F_{u}^{\prime \prime}(0)[z]^{2}=0
$$


Example 3 Let us consider Eq. 3 where $\varepsilon>0$ is sufficiently small parameter, $F: X \rightarrow X, X=L_{2}[0,1], x_{0}=0$. Mapping $F$ is infinitely differentiable on $X$ and moreover [12]

$$
\begin{gathered}
\left(F^{\prime}\left(x_{0}\right)[\xi]\right)(t)=\xi(t)-\frac{1}{\pi} \int_{0}^{2 \pi} \cos (t-\tau) \xi(\tau) d \tau \\
\left(F^{\prime \prime}\left(x_{0}\right)\left[\xi_{1}, \xi_{2}\right]\right)(t)=-\frac{2}{\pi} \sin t \int_{0}^{2 \pi} \sin \tau \cos \tau \xi_{1}(\tau) \xi_{2}(\tau) d \tau .
\end{gathered}
$$

Operator $F^{\prime}\left(x_{0}\right)$ is Fredholm of second kind with continuous kernel and $\operatorname{Ker} F^{\prime}\left(x_{0}\right)=\operatorname{span}\left\{u_{1}(t), u_{2}(t)\right\}$ where $u_{1}(t)=\frac{1}{\pi} \sin t, u_{2}(t)=\frac{1}{\pi} \cos t$. Then

$$
\operatorname{Im} F^{\prime}\left(x_{0}\right)=\left(\operatorname{Ker} F^{\prime}\left(x_{0}\right)\right)^{\perp} \neq X
$$

since $\cos t \notin \operatorname{Im} F^{\prime}\left(x_{0}\right),\left\langle u_{2}(t), \cos t\right\rangle=-1 \neq 0$, i.e. $F^{\prime}$ is non-surjective at $x_{0}=0$ (singular). Following the construction in Example 1 we obtain

$$
C^{j k}=c_{1}^{j k}=\left\langle u_{1}, F^{\prime \prime}\left(x_{0}\right)\left[u_{j}, u_{k}\right]\right\rangle, \quad j, k=1,2,
$$

$C^{11}=C^{22}=0, C^{12}=C^{21}=-\frac{1}{2}$ and corresponding quadratic form

$$
Q[z]^{2}=\sum_{j, k=1,2} C^{j k} z_{j} z_{k}=-z_{1} z_{2}
$$

is 2-regular along $h_{1}=(1,0), h_{2}=(0,1)$ (which belongs to $\left.\operatorname{Ker}^{2} Q[\cdot]^{2}\right)$ since the matrices $M(z)=\left(-z_{2},-z_{1}\right)$ are non-singular for $h_{1}, M\left(h_{1}\right)=(0,-1)$ and for $h_{2}, M\left(h_{2}\right)=(-1,0)$.

In next two examples we present mappings for which regularity conditions fails.

Example 4 Let us consider the following boundary value problem

$$
x^{\prime \prime}(t)+x(t)+x^{2}(t)=0, \quad x(0)=x(2 \pi)=0,
$$

where $x \in \mathcal{C}^{2}[0,2 \pi]$ and verify existence of the solution to this problem. In order to apply Theorem 2 introduce the mapping $F: X \rightarrow Y, F(x)=x^{\prime \prime}+x+x^{2}$, where $X=\left\{x \in \mathcal{C}^{2}[0,2 \pi]: x(0)=x(2 \pi)=0\right\}, Y=\mathcal{C}[0,2 \pi]$. Let $x_{0}=0$. Then

$$
Y_{1}=\operatorname{Im} F^{\prime}\left(x_{0}\right)=\left\{y \in Y: \int_{0}^{2 \pi} y(\tau) \sin \tau d \tau=0\right\} .
$$

Pay attention that $Y_{1} \neq Y, \operatorname{Ker} F^{\prime}\left(x_{0}\right)=\operatorname{span}(\sin t)=Y_{2}$,

$$
P_{2} y=2 \sin t \int_{0}^{2 \pi} \sin \tau y(\tau) d \tau, \quad y \in Y .
$$

Here $\operatorname{Ker}^{2} P_{2} F^{\prime \prime}\left(x_{0}\right)=\left\{x \in X: \int_{0}^{2 \pi} \sin \tau x^{2}(\tau) d \tau=0\right\}$, $\operatorname{Ker} F^{\prime}\left(x_{0}\right) \cap \operatorname{Ker}^{2} P_{2} F^{\prime \prime}\left(x_{0}\right)=\operatorname{span}\{\sin t\}$. 
The 2-regularity condition of $F$ along $h(t)=\sin t$ is equivalent to $\left.\operatorname{Im} P_{2} F^{\prime \prime}(0) h(t)\right|_{\operatorname{Ker} F^{\prime}(0)}=Y_{2}$. But

$$
P_{2} F^{\prime \prime}(0) h(t) \operatorname{Ker} F^{\prime}(0)=\operatorname{span} \sin t \int_{0}^{2 \pi} \sin ^{3} t d t=0 \neq Y_{2} .
$$

It means that the mapping $F$ is not 2-regular along $h(t)=\sin t$ at the point $x_{0}=0$. Example 5 Let us consider similar equation to (10), where its righthand side is equal to $\varepsilon \sin ^{2} t$, namely:

$$
x^{\prime \prime}(t)+x(t)+x^{2}(t)=\varepsilon \sin ^{2} t, \quad x(0)=x(2 \pi)=0,
$$

Here $F(x)=x^{\prime \prime}(t)+x(t)+x^{2}(t)-\varepsilon \sin ^{2} t, x_{0}=0$ and analogously to the above example the condition of 2-regularity of the mapping $F$ along $h(t)=\sin t$ at $x_{0}=0$ fails.

Below we give an example of 3-regular mapping, which is useful in applications.

Example 6 Let us consider the equation of beam deformation which appears often in applications

$$
F(x)=x^{\prime \prime}(t)+x(t)+\mu x^{3}(t)+\varepsilon \sin t=0, \quad x(0)=x(2 \pi)=0,
$$

where $\mu \neq 0$. For $x_{0}=0$ the operator $F^{\prime}\left(x_{0}\right)$ is singular i.e. the equation

$$
x^{\prime \prime}(t)+x(t)-\varepsilon \sin t=0, \quad x(0)=x(2 \pi)=0,
$$

has no solutions. We have $\operatorname{Ker} F^{\prime}\left(x_{0}\right) \cap \operatorname{Ker}^{2} P_{2} F^{\prime \prime}\left(x_{0}\right)=\operatorname{span}(\sin t)$. Since $\operatorname{Ker}^{2} P_{2} F^{\prime \prime}\left(x_{0}\right)=X=\left\{x \in \mathcal{C}^{2}[0,2 \pi]: x(0)=x(2 \pi)=0\right\}$, we omit $\operatorname{Ker}^{2} P_{2} F^{\prime \prime}\left(x_{0}\right)$ in the sequel. But for $h(t)=\sin t, 3$-factor operator

$$
\left\{F^{\prime}\left(x_{0}\right)+P_{3} F^{\prime \prime \prime}\left(x_{0}\right) h^{2}(t)\right\}(\cdot)=(\cdot)^{\prime \prime}+(\cdot)+3 \mu \sin t \int_{0}^{2 \pi} \sin \tau \cdot h^{2}(\tau)(\cdot) d \tau
$$

is surjective, i.e. the mapping $F(\cdot)$ is 3-regular along $h(t)=\sin t$ at the point $x_{0}=0$.

The following theorem gives a description of a solution set in singular (degenerate) case.

Theorem (Generalized Lyusternik Theorem) [5, 11] Let $X$ and $Y$ be Banach spaces and $U$ be a neighborhood of $x_{0} \in X$. Assume that $F: X \rightarrow Y, F \in C^{p}(U)$ is p-regular at $x_{0}$. Then

$$
T_{1} M\left(x_{0}\right)=\operatorname{Ker}^{p} \Psi_{p}[x]^{p} .
$$

We shall give two auxiliary lemmas. The first of these lemmas is a "multivalued" generalization of the contraction mapping principle and is independent interest. By $\operatorname{dist}_{\mathrm{H}}\left(A_{1}, A_{2}\right)$ we mean the Hausdorff distance between sets $A_{1}$ and $A_{2}$.

Lemma 1 (Contraction Multimapping Principle) [1, 8, 10, 14] Let $Z$ be a complete metric space with distance $\rho$. Assume that we are given a multimapping

$$
\Phi: U_{v}\left(z_{0}\right) \rightarrow 2^{Z}
$$


on a ball $U_{v}\left(z_{0}\right)=\left\{z: \rho\left(z, z_{0}\right)<v\right\} \quad(v>0)$ where sets $\Phi(z)$ are non-empty and closed for any $z \in U_{v}\left(z_{0}\right)$. Further, assume that there exists a number $\theta, 0<\theta<1$ such that

1. $\operatorname{dist}_{\mathrm{H}}\left(\Phi\left(z_{1}\right), \Phi\left(z_{2}\right)\right) \leq \theta \rho\left(z_{1}, z_{2}\right)$ for any $z_{1}, z_{2} \in U_{v}\left(z_{0}\right)$

2. $\rho\left(z_{0}, \Phi\left(z_{0}\right)\right)<(1-\theta) v$.

Then, for every number $\varepsilon_{1}$ which satisfies the inequality

$$
\rho\left(z_{0}, \Phi\left(z_{0}\right)\right)<\varepsilon_{1}<(1-\theta) v,
$$

there exists $z \in B_{\varepsilon_{1} /(1-\theta)}\left(z_{0}\right)=\left\{\omega: \rho\left(\omega, z_{0}\right) \leq \varepsilon_{1} /(1-\theta)\right\}$ such that

$$
z \in \Phi(z)
$$

Lemma $2[1,10]$ Let $\Lambda \in \mathcal{L}(X, Y)$. We set

$$
C(\Lambda)=\sup _{\|y\|=1} \inf \{\|x\|: x \in X, \Lambda x=y\} .
$$

If $\operatorname{Im} \Lambda=Y$, then $C(\Lambda)<\infty$.

Lemma 3 (Mean Value Theorem) $[1,10]$ Let $U$ be an open subset of the Banach space $X$ such that $[a, b] \in U$. If $f: U \rightarrow Y$ and $f \in \mathcal{C}^{1}(U)$ then

$$
\|f(b)-f(a)-\Lambda(b-a)\| \leq \sup _{\xi \in[a, b]}\left\|f^{\prime}(\xi)-\Lambda\right\| \cdot\|a-b\|,
$$

for any $\Lambda \in \mathcal{L}(X, Y)$.

For better understanding of the main result of this paper we consider more simple analogous result in non-degenerate (regular) case.

\section{Regular Case}

We quote one modification of the theorem on existence of solutions to the equations with non-degenerate mappings (see in [15]). The essential idea of the proof in the singular case is based on the similar construction to the one in the regular case.

Let us consider the mapping $F: X \rightarrow Y$. We are interested in the existence of such a point $x^{*}$ that $F\left(x^{*}\right)=0$. Throughout this section we assume that an initial point $x_{0}$ is given such that $F(x)$ is regular at $x_{0}$, i.e. $F^{\prime}\left(x_{0}\right) X=Y$ and that $\left\{F^{\prime}\left(x_{0}\right)\right\}^{-1}$ is a multivalued mapping. Moreover, let $\operatorname{Ker} F^{\prime}\left(x_{0}\right) \neq\{0\}$ and $U_{\varepsilon}\left(x_{0}\right)=\left\{x \in X:\left\|x-x_{0}\right\| \leq \theta\right\}$ where $0<\theta<1$.

Theorem 2 Let $F \in \mathcal{C}^{2}(X), \quad\left\|F\left(x_{0}\right)\right\|=\delta, \quad F^{\prime}\left(x_{0}\right): X \stackrel{\text { on }}{\rightarrow} Y, \quad\left\|\left\{F^{\prime}\left(x_{0}\right)\right\}^{-1}\right\|=\eta$ and $\sup _{x \in U_{v}\left(x_{0}\right)}\left\|F^{\prime \prime}(x)\right\|=c<\infty, \quad h \in \operatorname{Ker} F^{\prime}\left(x_{0}\right),\|h\|=1$. If there exists $\omega, 0<\omega<\frac{1}{2} v$ such that the following inequalities

1. $\delta \cdot \eta \leq \frac{\omega}{8}$,

2. $\omega \cdot c \cdot \eta \leq \frac{1}{6}$ 
hold then the equation $F(x)=0$ has a solution $x^{*}=x_{0}+\omega h+\bar{x}(\omega) \in U_{v}\left(x_{0}\right)$ and $\|\bar{x}(\omega)\| \leq \frac{1}{2} \omega$.

Proof Define a multivalued mapping

$$
\begin{gathered}
\Phi: U_{v}(0) \rightarrow 2^{X}, \quad U_{v}(0) \subset X, \\
\Phi(x)=x-\left\{F^{\prime}\left(x_{0}\right)\right\}^{-1}\left(F\left(x_{0}+\omega h+x\right)\right), \quad x \in U_{\omega}(0) .
\end{gathered}
$$

The sets $\Phi(x)$ are nonempty and closed for any $x \in U_{\omega}(0)$. It follows from the assumption that $F^{\prime}\left(x_{0}\right)$ is a surjection and that the sets $\left\{F^{\prime}\left(x_{0}\right)\right\}^{-1}(y)$ for $y \in Y$, are linear manifolds parallel to $\operatorname{Ker} F^{\prime}\left(x_{0}\right)$.

We shall show that

$$
\operatorname{dist}_{\mathrm{H}}\left(\Phi\left(s_{1}\right), \Phi\left(s_{2}\right)\right) \leq \frac{1}{2}\left\|s_{1}-s_{2}\right\|, \text { for any } s_{1}, s_{2} \in U_{\omega}(0) .
$$

Indeed, by Lemmas 2 and 3 we have

$$
\operatorname{dist}_{\mathrm{H}}\left(\Phi\left(s_{1}\right), \Phi\left(s_{2}\right)\right) \leq 3 \eta \cdot c \cdot \omega\left\|s_{1}-s_{2}\right\|
$$

and (14) is proved.

Moreover we have

$$
\begin{aligned}
\varrho(\Phi(0), 0) & =\inf \left\{\|z\|: F^{\prime}\left(x_{0}\right) z=-F\left(x_{0}+\omega h\right)\right\} \leq \delta\left\|F\left(x_{0}+\omega h\right)\right\| \\
& \leq \delta \| F\left(x_{0} \|+\frac{1}{2} \eta \cdot c \cdot \omega^{2}<\frac{1}{8} \omega+\frac{1}{12} \omega<\frac{1}{4} \omega .\right.
\end{aligned}
$$

It means that all the assumptions of Lemma 1 are fulfilled and there exists $\bar{x}(\omega) \in \Phi(\bar{x}(\omega))$. Consequently, $\bar{x}(\omega) \in \bar{x}(\omega)+\left\{F^{\prime}\left(x_{0}\right)\right\}^{-1}\left(-F\left(x_{0}+\omega h+\bar{x}(\omega)\right)\right)$. Hence $0 \in\left\{F^{\prime}\left(x_{0}\right)\right\}^{-1}\left(-F\left(x_{0}+\omega h+\bar{x}(\omega)\right)\right.$ and $F\left(x_{0}+\omega h+\bar{x}(\omega)\right)=0$ or $F\left(x^{*}\right)=0$. From (15), $\|\bar{x}(\omega)\| \leq \frac{1}{2} \omega$ or $\|\omega h+\bar{x}(\omega)\| \leq \frac{1}{2} v+\frac{1}{4} v<v$, i.e. $x^{*} \in U_{v}\left(x_{0}\right)$.

Remark 2 It is easy to see, that if in the condition 1 of Theorem 2 we assume that $\delta \cdot \eta \leq \omega^{1+\sigma}, \sigma>0$, then we obtain $\|\bar{x}(\omega)\|=o(\omega)$.

Remark 3 (Sequential Regularity) The assumptions of Theorem 2 may be weakened if, instead of the mapping $\Phi(x)$, we consider the following sequence $x_{0}=0$, $x_{n+1} \in x_{n}-\left\{F^{\prime}\left(x_{0}\right)\right\}^{-1} F\left(x_{0}+\omega h+x_{n}\right), n=1,2, \ldots$ And consequently, instead of surjection of the mapping $F^{\prime}\left(x_{0}\right)$ we can assume that $\operatorname{Im} F^{\prime}\left(x_{0}\right)=W \subset Y$, where $W$ is some closed subspace of $Y$ and $F\left(x_{0}+\omega h+x_{n}\right) \subset W, n=0,1, \ldots$

\section{4 p-Regular (Singular) Case}

In the previous paper [15] we have considered the case of the trivial $p$-kernel of $p$ order derivatives of the mapping $F(x)$ at the initial point $x_{0}$ (for completely degenerate case). For these purposes we required much stronger assumptions on the mapping $F(x)$ such as invertibility of nonlinear operator $F^{(p)}\left(x_{0}\right)[\cdot]^{p}$, uniform boundedness of $\left\{F^{(p)}\left(x_{0}\right)[\cdot]^{p}\right\}^{-1}$ and so on. In nontrivial $p$-kernel case, $\operatorname{Ker}^{p} F^{(p)}\left(x_{0}\right) \neq\{0\}$ these assumptions we omit and remain only assumptions of $p$-regularity of the mapping 
$F(x)$ along some element belonging to $p$-kernel of $F^{(p)}\left(x_{0}\right)$ and some quantitative assumptions like in the case of trivial $p$-kernel of mapping $F^{(p)}\left(x_{0}\right)$.

Let us introduce the following notations and assumptions.

$$
\begin{aligned}
& \delta=\left\|F\left(x_{0}\right)\right\|, \\
& h \in \bigcap_{k=1}^{p} \operatorname{Ker}^{k} f_{k}^{(k)}\left(x_{0}\right) \\
& \eta=\left\|\Lambda_{h}^{-1}\right\|<\infty, \quad\|h\|=1, \\
& c=\max _{k=1, \ldots, p} \sup _{x \in U_{v}\left(x_{0}\right)}\left\|f_{k}^{(k+1)}(x)\right\|, \\
& d=4 \max _{k=1, \ldots, p} \frac{1}{(k-1) !}\left\|f_{k}^{(k)}\left(x_{0}\right)\right\|, \\
& \alpha=\min \left\{\frac{3}{4 p+2 \eta}, \min _{k=1, \ldots ., p} \frac{\left\|f_{k}^{(k)}\left(x_{0}\right)\right\|}{(k-1) !}\right\} .
\end{aligned}
$$

Now we can state the main result.

Theorem 3 Let $F: X \rightarrow Y$, and $X, Y-$ Banach spaces, $F \in \mathcal{C}^{p+1}(X)$. Assume that there exists $h \in \bigcap_{k=1}^{p} \operatorname{Ker}^{k} f_{k}^{(k)}\left(x_{0}\right),\|h\|=1$ such that $F$ is $p$-regular mapping at $x_{0}$ along $h$.

If there exists $\omega, 0<\omega<\frac{1}{2} v, v \in(0,1)$, such that the inequalities

1. $\eta \delta \leq \alpha \frac{\omega^{p}}{2 p d}$,

2. $\frac{4^{p+2}}{3} c \omega \eta \leq \frac{1}{2}$,

hold, then the equation $F(x)=0$ has a solution $x^{*}=x_{0}+\omega h+\bar{x}(\omega) \in U_{v}\left(x_{0}\right)$ and $\|\bar{x}(\omega)\| \leq \frac{1}{2} \omega$.

Remark 4 The following conditions are equivalent:

1. $F$ satisfies $p$-regularity condition at $x_{0}$ along $h$.

2. Operator $\Lambda_{\omega h}$ is surjective for any $\omega \neq 0$.

3. For $h$ condition (18) holds.

4. $\left\|\Lambda_{\omega h}^{-1} y\right\| \leq \eta\left(1+\frac{1}{\omega}+\frac{1}{w^{2}}+\ldots+\frac{1}{\omega^{p-1}}\right)$ for $\omega \neq 0$.

Proof of Theorem 3 Similarly like in the regular case let us consider a multivalued mapping

$$
\Phi_{\omega h}(x): U_{v}(0) \rightarrow Y
$$

such that

$$
\Phi_{\omega h}(x)=x-\Lambda_{\omega h}^{-1}\left\{f_{1}\left(x_{0}+\omega h+x\right), \ldots, f_{p}\left(x_{0}+\omega h+x\right)\right\},
$$

where $x \in U_{v}(0)$.

Like in regular case, the assumptions of contraction multimapping principle hold for $\Phi_{\omega h}(x)$. Indeed, the sets $\Phi_{\omega h}(x)$ are non-empty because $\Lambda_{\omega h}$ is a surjection for any $x \in U_{v}(0)$. 
Moreover, for any $y \in Y_{1} \times \ldots \times Y_{p}$ the sets $\Lambda_{\omega h}^{-1}(y)$ are linear manifolds parallel to $\operatorname{Ker} \Lambda_{\omega h}$, and hence the sets $\Phi_{\omega h}(x)$ are closed for any $x \in U_{v}(0)$.

We prove that

$$
\operatorname{dist}_{\mathrm{H}}\left(\Phi_{\omega h}\left(x_{1}\right), \Phi_{\omega h}\left(x_{2}\right)\right) \leq \frac{1}{2}\left\|x_{1}-x_{2}\right\|
$$

for $x_{1}, x_{2} \in U_{v}(0)$ such that $\left\|x_{i}\right\| \leq \frac{\omega}{R}, \quad i=1,2$, where $R=\max _{k=1, \ldots, p} R_{k}, \quad$ and $R_{k}=\frac{\left\|f_{k}^{(k)}\left(x_{0}\right)\right\|}{\alpha(k-1) !}$.

Let us point out that $R_{k} \geq 1, \forall k=1, \ldots, p$ and it implies that $R \geq 1$ and $\left\|x_{i}\right\| \leq \frac{\omega}{R} \leq \omega$. Let $s_{1}=x_{0}+t h+x_{1}, s_{2}=x_{0}+t h+x_{2}$. Then

$$
\begin{aligned}
& \operatorname{dist}_{\mathrm{H}}\left(\Phi_{\omega h}\left(x_{1}\right), \Phi_{\omega h}\left(x_{2}\right)\right)=\inf \left\{\left\|z_{1}-z_{2}\right\|: z_{j} \in \Phi_{\omega h}\left(x_{j}\right), j=1,2\right\} \\
& =\inf \left\{\left\|z_{1}-z_{2}\right\|: \Lambda_{\omega h}\left(z_{j}\right)=\Lambda_{\omega h}\left(x_{j}\right)-\left(f_{1}\left(s_{j}\right), \ldots, f_{p}\left(s_{j}\right)\right), j=1,2\right\} \\
& \leq \inf \left\{\|z\|: \Lambda_{\omega h}(z)=\Lambda_{\omega h}\left(x_{1}-x_{2}\right)-\left(f_{1}\left(s_{1}\right)-f_{1}\left(s_{2}\right), \ldots, f_{p}\left(s_{1}\right)-f_{p}\left(s_{2}\right)\right)\right\} \\
& =\inf \left\{\|z\|: \Lambda_{\omega h}(z)=\left(\Lambda_{\omega h, 1}\left(x_{1}-x_{2}\right)-f_{1}\left(s_{1}\right)+f_{1}\left(s_{2}\right), \ldots\right.\right. \\
& \left.\left.\quad \ldots, \frac{1}{\omega^{p-1}}\left(\Lambda_{\omega h, p}\left(x_{1}-x_{2}\right)-f_{p}\left(s_{1}\right)+f_{p}\left(s_{2}\right)\right)\right)\right\} \\
& \leq \inf \left\{\|z\|: z=\Lambda_{\omega h}^{-1}\left(\Lambda_{\omega h, 1}\left(x_{1}-x_{2}\right)-f_{1}\left(s_{1}\right)+f_{1}\left(s_{2}\right), \ldots\right.\right. \\
& \left.\left.\quad \ldots, \frac{1}{\omega^{p-1}}\left(\Lambda_{\omega h, p}\left(x_{1}-x_{2}\right)-f_{p}\left(s_{1}\right)+f_{p}\left(s_{2}\right)\right)\right)\right\} \\
& \leq \eta \cdot \sum_{k=1}^{p} \frac{1}{\omega^{k-1}}\left\|f_{k}\left(s_{1}\right)-f_{k}\left(s_{2}\right)-\Lambda_{\omega h, k}\left(x_{1}-x_{2}\right)\right\| .
\end{aligned}
$$

Taking into account Lemma 3 we have

$$
\begin{gathered}
\left\|f_{k}\left(s_{1}\right)-f_{k}\left(s_{2}\right)-\Lambda_{\omega h, k}\left(x_{1}-x_{2}\right)\right\| \leq \sup _{\theta \in[0,1]}\left\|f_{k}^{\prime}\left(s_{k}+\theta\left(x_{1}-x_{2}\right)\right)-\Lambda_{\omega h, k}\right\| \\
\cdot\left\|x_{1}-x_{2}\right\| .
\end{gathered}
$$

As $f_{k}(x)$ is completely degenerate up to the order $k$ we obtain the following Taylor expansion

$$
\begin{aligned}
f_{k}^{\prime}\left(s_{2}+\theta\left(x_{1}-x_{2}\right)\right)= & f_{k}^{\prime}\left(x_{0}\right)+\ldots+\frac{f_{k}^{(k)}\left(x_{0}\right)\left[s_{2}-x_{0}+\theta\left(x_{1}-x_{2}\right)\right]^{k-1}}{(k-1) !} \\
& +\omega_{k}\left(\omega h, x_{1}, x_{2}, \theta\right) \\
= & \frac{f_{k}^{(k)}\left(x_{0}\right)\left[s_{2}-x_{0}+\theta\left(x_{1}-x_{2}\right)\right]^{k-1}}{(k-1) !} \\
& +\omega_{k}\left(\omega h, x_{1}, x_{2}, \theta\right),
\end{aligned}
$$




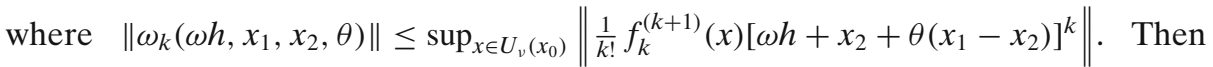
from the assumption (21) we have $\left\|\omega h+x_{2}+\theta\left(x_{1}-x_{2}\right)\right\| \leq 4 \omega$. This and previous formulae imply

$$
\left\|\omega_{k}\left(\omega h, x_{1}, x_{2}, \theta\right)\right\| \leq \frac{1}{k !} c\left\|\omega h+x_{2}+\theta\left(x_{1}-x_{2}\right)\right\|^{k} \leq \frac{1}{k !} 4^{k} \omega^{k} c
$$

and

$$
\begin{aligned}
& f_{k}^{(k)}\left(x_{0}\right)\left[s \omega h+x_{2}+\theta\left(x_{1}-x_{2}\right)\right]^{k-1} \\
& =\sum_{n=0}^{k-1}\left(\begin{array}{c}
k-1 \\
n
\end{array}\right) f_{k}^{(k)}\left(x_{0}\right)[\omega h]^{k-1-n}\left[x_{2}+\theta\left(x_{1}-x_{2}\right)\right]^{n} \\
& =f_{k}^{(k)}\left(x_{0}\right)[\omega h]^{k-1}+\sum_{n=1}^{k-1}\left(\begin{array}{c}
k-1 \\
n
\end{array}\right) f_{k}^{(k)}\left(x_{0}\right)[\omega h]^{k-1-n}\left[x_{2}+\theta\left(x_{1}-x_{2}\right)\right]^{n} .
\end{aligned}
$$

Moreover

$$
\left\|x_{2}+\theta\left(x_{1}-x_{2}\right)\right\| \leq 3 \frac{\omega}{R} \leq \frac{3 \omega}{R_{k}} .
$$

Taking into account the definition of $R_{k}$, we get

$$
\begin{aligned}
& \left\|\sum_{n=1}^{k-1}\left(\begin{array}{c}
k-1 \\
n
\end{array}\right) f_{k}^{(k)}\left(x_{0}\right)[\omega h]^{k-1-n}\left[x_{2}+\theta\left(x_{1}-x_{2}\right)\right]^{n}\right\| \\
& \leq\left\|f_{k}^{(k)}\left(x_{0}\right)\right\| \cdot \sum_{n=1}^{k-1}\left(\begin{array}{c}
k-1 \\
n
\end{array}\right) \omega^{k-1-n}(3 \omega)^{n} / R_{k}^{n} \\
& \leq\left\|f_{k}^{(k)}\left(x_{0}\right)\right\| \cdot \omega^{k-1} \cdot 4^{k-1} / R_{k} \leq 4^{k}(k-1) ! \omega^{k-1} \alpha .
\end{aligned}
$$

Now, inserting (24)-(28) into (23) we obtain

$$
\left\|f_{k}\left(s_{1}\right)-f_{k}\left(s_{2}\right)-\Lambda_{\omega h, k}\left(x_{1}-x_{2}\right)\right\| \leq 4^{k} \omega^{k-1}\left(\alpha+\frac{c \omega}{k !}\right) \cdot\left\|x_{1}-x_{2}\right\| .
$$

Hence

$$
\begin{aligned}
\operatorname{dist}_{\mathrm{H}}\left(\Phi_{\omega h}\left(x_{1}\right), \Phi_{\omega h}\left(x_{2}\right)\right) & \leq \eta \cdot \sum_{k=1}^{p} \frac{1}{\omega^{k-1}} 4^{k} \omega^{k-1}(\alpha+c \omega)\left\|x_{1}-x_{2}\right\| \\
& =\eta \sum_{k=1}^{p} 4^{k}(\alpha+c \omega)\left\|x_{1}-x_{2}\right\| \\
& \leq \frac{\eta \cdot 4^{p+1}}{3}(\alpha+c \omega)\left\|x_{1}-x_{2}\right\| \leq \frac{1}{2}\left\|x_{1}-x_{2}\right\|
\end{aligned}
$$

(by virtue of (21) and assumption 2), which proves (22). 
Now let us estimate $\left\|\Phi_{\omega h}(0)\right\|$. We have

$$
\begin{aligned}
\left\|\Phi_{\omega h}(0)\right\| & =\inf \left\{\|z\|: z \in \Phi_{\omega h}(0)\right\}=\eta \sum_{k=1}^{p} \frac{1}{\omega^{k-1}}\left\|f_{k}\left(x_{0}\right)-\omega_{k}\left(x_{0}, \omega h\right)\right\| \\
& \leq \eta \sum_{k=1}^{p}\left(\frac{1}{\omega^{k-1}}\left\|f_{k}\left(x_{0}\right)\right\|+\frac{1}{\omega_{k-1}} \frac{c \omega^{k+1}}{(k-1) !}\right) \\
& \leq \eta \sum_{k=1}^{p}\left(\frac{1}{\omega^{k-1}}\left\|f_{k}\left(x_{0}\right)\right\|+\frac{c \omega^{2}}{(k-1) !}\right) \\
& \leq \eta \cdot p \frac{\delta}{\omega^{p-1}}+p \cdot \eta \cdot c \cdot \omega^{2} .
\end{aligned}
$$

It follows from assumption 1 , that $\frac{\eta p \delta}{\omega^{p-1}} \leq \frac{\omega}{8 R}$, since $\frac{\alpha}{d} \leq \frac{1}{4}$. From assumption 2) we obtain $p \eta c \cdot \omega^{2} \leq \frac{\omega}{8 R}$, and finally $\left\|\Phi_{\omega h}(0)\right\| \leq \frac{\omega}{4 R}$.

From the above and from (22) we obtain that for the mapping $\Phi_{\omega h}(x)$ all the assumptions of contraction multimapping principle hold with $\theta=\frac{1}{2}, \varepsilon_{1}=\frac{\omega}{4 R}$ and hence there exist an element $\bar{x}(\omega) \in \Phi_{\omega h}(\bar{x}(\omega))$ or $F\left(x_{0}+\omega h+\bar{x}(\omega)\right)=0$ such that $\|\bar{x}(\omega)\| \leq \frac{\omega}{2 R} \leq \frac{\omega}{2}$. It follows that $x_{0}+\omega h+\bar{x}(\omega)$ is the solution to the Eq. 1.

Remark 5 As in the regular case if we assign $\alpha=\omega^{\sigma}, \sigma>0$ then we obtain

$$
\|\bar{x}(\omega)\|=o(\omega) .
$$

Remark 6 (Sequential $p$-Regularity) Similarly, like in the regular case the assumptions of Theorem 3 can be weakened if we consider the sequence: $x_{0}=0, x_{n+1} \in x_{n}-\Lambda_{\omega h}^{-1}\left(f_{1}\left(x_{0}+\omega h+x_{n}\right), \ldots, f_{p}\left(x_{0}+\omega h+x_{n}\right)\right), n=1,2, \ldots$, instead of the mapping $\Phi_{\omega h}(x)$. Moreover, instead of $p$-regularity condition of the mapping $F$ at the point $x_{0}$ we can assume $p$-regularity of $F$ at $\left\{x_{n}\right\}$ i.e. instead of operator $\Lambda_{h}$ surjectivity we can require that $\operatorname{Im} \Lambda_{h}=W \subset Y$, where $W$ is some closed subspace of $Y$ and $F\left(x_{0}+\omega h+x_{n}\right) \in W, n=0,1, \ldots$

We conclude the discussion with the examples from the beginning of the hereby paper, which serve now to illustrate the basic idea of Theorem 3.

\section{Applications and Examples}

We continue consideration of Examples 1-5 and we verify for them assumptions of Theorem 3.

Example 1 As it was shown earlier the main assumption of Theorem 3, that is $p$ regularity condition of the mapping $F(u, \varepsilon)$, is fulfilled at the point $u_{0}=0, \varepsilon_{0}=0$. We calculate $\delta=\|F(0,0)\|=0$. It means that assumption 1 of Theorem 3 holds. Second assumption is true for $v>0$ sufficiently small if

$$
\omega \leq \frac{3}{2 \cdot 4^{p+2} \cdot c \cdot \eta} \quad \text { and } \omega<\frac{1}{2} \nu .
$$


It means that there exists a solution of Eq. 6 of the following form

$$
u^{*}(\omega)=u^{*}(\varepsilon)=\varepsilon\left(\bar{z}_{1} u_{1}+\bar{z}_{2} u_{2}\right)+\bar{u}(\varepsilon)
$$

where $\|\bar{u}(\varepsilon)\|=o(\varepsilon)$.

Example 2 In Section 2 it was shown that the mapping $F(u)$ is $p$-regular at the point $u_{0}=0$ if

$$
\text { Rank } P_{2} F^{\prime \prime}(0)[z]=\operatorname{Rank} M(z)=l
$$

for $z=z_{1} u_{1}+\ldots+z_{l} u_{l} \in \operatorname{Ker}^{2} P_{2} F_{u}^{\prime \prime}(0)$. Here $l=2$. Assumption 1 of Theorem 3 holds, because we have $\delta=\|F(0)\|=\varepsilon, \omega \sim \sqrt{\varepsilon}$ and $\varepsilon$ is sufficiently small.

Assumption 2 we obtain taking $\omega<\min \left\{\frac{3}{2 \cdot 4^{p+2} \cdot c \cdot \eta}, \frac{1}{2} v\right\}$. Hence there exists a solution to (9) of the form

$$
u(y, \varepsilon)=\sqrt{\varepsilon}\left(\bar{z}_{1} u_{1}+\bar{z}_{2} u_{2}\right)+\bar{u}(y, \varepsilon),
$$

where $\|\bar{u}(y, \varepsilon)\|=o(\sqrt{\varepsilon})$.

Example 3 It was verified in Section 2 that the mapping $F(x)$ is 2-regular at the point $x_{0}=0$ along $h_{1}=(1,0), h_{2}=(0,1)$. Moreover $\delta=\|F(0)\|=\frac{\varepsilon}{\sqrt{\pi}}$. Hence, for

$$
\sqrt{\frac{4 \varepsilon \cdot \eta \cdot d}{\sqrt{\pi} \cdot \alpha}} \leq \omega<\frac{1}{2} v
$$

and for sufficiently small $\varepsilon>0$ assumptions 1 and 2 of Theorem 3 hold. It means that there exist solutions $x(t, \varepsilon)$ to Eq. 3 of the following form

$$
\begin{aligned}
& x_{1}(t, \varepsilon)=\frac{\sqrt{\varepsilon}}{\sqrt{\pi}} \cos t+\bar{x}_{1}(t, \varepsilon) \\
& x_{2}(t, \varepsilon)=\frac{\sqrt{\varepsilon}}{\sqrt{\pi}} \sin t+\bar{x}_{2}(t, \varepsilon),
\end{aligned}
$$

where $\left\|\bar{x}_{1,2}(t, \varepsilon)\right\|=o(\sqrt{\varepsilon})$.

Example 4 We have shown already that $F(x)$ is not $p$-regular mapping at the point $x_{0}$ along $h(t)=\sin t \in \operatorname{Ker} F^{\prime}\left(x_{0}\right) \cap \operatorname{Ker}^{2} P_{2} F^{\prime \prime}\left(x_{0}\right)$.

Although assumption of Theorem 3 fails, the assumptions of Remark 6 are fulfilled because considered mapping is sequentially 2-regular. Indeed, let $W=\operatorname{Im} \Lambda_{h, 1}$, where $\Lambda_{h, 1}=\ddot{(\cdot)}+1(\cdot)$. Then $F\left(x_{0}+\omega h+x_{n}\right) \in \operatorname{Im} W$ for $x_{1}=0$, $x_{n+1}=x_{n}-\Lambda_{h, 1}^{-1} F\left(x_{0}+\omega h+x_{n}\right)$. Let us consider for instance $x_{2}$. We have

$$
\ddot{x}_{2}+x_{2}+F\left(x_{0}+\omega h\right)=\ddot{x}_{2}+x_{2}+\omega^{2} \sin ^{2} t=0 .
$$

Since $\int_{0}^{2 \pi} \omega^{2} \sin ^{3} \tau d \tau=0$. This equation has a solution $x_{2}(t)$ such that $x_{2}(0)=x_{2}(2 \pi)=0$. Similarly we can calculate elements $x_{n}$, for $n>2$. 
Both assumptions of Theorem 3 are fulfilled trivially for $0<\omega<1 / 2 v, \omega \leq \frac{3}{2 \cdot 4^{4} \cdot \cdot \cdot \eta}$, because $\delta=\|F(0)\|=0$ and $\eta=\left\|\Lambda_{h, 1}^{-1}\right\|>0$. It means that if we substitute $\omega^{\sigma}, \sigma>0$ (see Remark 5) such that $\omega>0$ for $\alpha$ in assumption 1 of Theorem 3, then there exists a solution to (10) of the following form

$$
x^{*}(t)=\omega \cdot \sin t+\bar{x}(\omega, t), \quad \text { where } \quad\|\bar{x}(\omega, t)\|=o(\omega) .
$$

Example 5 Similarly like in the previous example, mapping $F(x)$ from (11) is sequentially 2-regular at the point $x_{0}=0$ along $h(t)=\sin t$. Assumptions 1 and 2 of Theorem 3 are satisfied because $\delta=\|F(0)\|=\varepsilon>0$. If we take into account $\alpha=\omega^{\sigma}$, $\sigma>0$, then for such $\delta=\varepsilon$ that $\eta \varepsilon \leq \frac{\omega^{2+\sigma}}{4 d}$ we obtain that there exists a solution $x^{*}(t, \varepsilon)$ to the Eq. 11 of the following form

$$
x^{*}(t, \varepsilon)=\sqrt{\varepsilon} \sin t+\bar{x}(t, \varepsilon),
$$

where $\|\bar{x}(t, \varepsilon)\|=o(\sqrt{\varepsilon})$. In fact $x^{*}(t, \varepsilon)=\sqrt{\varepsilon} \sin t$ is a solution of (11).

Acknowledgements Research of the second author is supported by the Russian Foundation for Basic Research Grant 08-01-00619 and the Council for the State Support of Leading Scientific Schools Grant 4096.2010.1.

Open Access This article is distributed under the terms of the Creative Commons Attribution Noncommercial License which permits any noncommercial use, distribution, and reproduction in any medium, provided the original author(s) and source are credited.

\section{References}

1. Alexeev, V.M., Tihomirov, V.M., Fomin, S.V.: Optimal Control. Consultants Bureau, New York (1987)

2. Belash, K.N., Tret'yakov, A.A.: Methods for solving degenerate problems. USSR Comput. Math. Math. Phys. 28, 90-94 (1988)

3. Bereznev, V.A., Karmanov, V.G., Tret'yakov, A.A.: The Stable Methods for Solving Extremal Problems with Approximate Data. Science Council for Complex Problem "Cybernetics", Moscow (1987). In Russian

4. Brezhneva, O.A., Tret'yakov, A.A.: Solvability of the Cauchy problem for a first-order partial differential equations in the degenerate case. Differ. Equ. 38(2), 228-234 (2002)

5. Brezhneva, O.A., Tret'yakov, A.A.: Optimality conditions for degenerate extremum problems with equality constraints. SIAM J. Control Optim. 42, 729-745 (2003)

6. Brezhneva, O.A., Tret'yakov, A.A.: The $p$-th order optimality conditions for nonregular optimization problems. Dokl. Math. 77(2), 163-165 (2008)

7. Brezhneva, O.A., Tret'yakov, A.A., Marsden, J.E.: Higher-order implicit function theorem and degenerate nonlinear boundary-value problems. Commun. Pure Appl. Anal. 2, 425-445 (2003)

8. Covitz, H., Nadler Jr., S.B.: Multi-valued contraction mappings in generalized metric spaces. Isr. J. Math. 8(1), 5-11 (1970)

9. Demidowich, B.P., Maron, I.A.: Basics of Computational Mathematics. Nauka, Moscow (1973). In Russian

10. Ioffe, A.D., Tihomirov, V.M.: Theory of Extremal Problems. Studies in Mathematics and its Applications. North-Holland, Amsterdam (1979)

11. Izmailov, A.F., Tret'yakov, A.A.: Factor-Analysis of Nonlinear Mappings. Nauka, Moscow (1994). In Russian

12. Izmailov, A.F., Tret'yakov, A.A.: 2-Regular Solutions of Nonlinear Problems. Theory and Numerical Methods. Fizmatlit, Moscow (1999). In Russian

13. Korneva, I.T., Tret'yakov, A.A.: Application of the factor-analysis to the calculus of variations. In: Proceedings of Simulation and Analysis in Problems of Decision-Making Theory, pp. 144162. Computing Center of Russian Academy of Sciences, Moscow (2002). In Russian 
14. Nadler, Jr., S.B.: Multi-valued contraction mapping. Not. Am. Math. Soc. 14, 930 (1967)

15. Prusińska, A., Tret'yakov, A.A.: A remark on the existence of solutions to nonlinear equations with degenerate mappings. Set-Valued Anal. 16, 93-104 (2008)

16. Tret'yakov, A.A.: Necessary and sufficient conditions for optimality of $p$-th order. Control and Optimization, pp. 28-35. MSU, Moscow (1983). In Russian

17. Tret'yakov, A.A.: Necessary and sufficient conditions for optimality of $p$-th order. Comput. Math. Math. Phys. 24, 123-127 (1984)

18. Tret'yakov, A.A.: The implicit function theorem in degenerate problems. Russ. Math. Surv. 42, 179-180 (1987)

19. Tret'yakov, A.A., Marsden, J.E.: Factor-analysis of nonlinear mappings: $p$-regularity theory. Commun. Pure Appl. Math. 2, 425-445 (2003) 